\title{
OPTICAL PARAMETRIC INTERACTIONS IN DISTRIBUTED MAGNETO-OPTICAL BRAGG GRATINGS
}

\author{
FREDRIK JONSSON \\ EPSRC Nanophotonics Portfolio Centre, \\ School of Physics and Astronomy, University of Southampton, \\ SO17 1BJ Southampton, United Kingdom \\ CHRISTOS FLYTZANIS \\ Laboratoire Pierre Aigrain, Ecole Normale Superieure, \\ 24 rue Lhomond, \\ 75231 Paris Cedex 05, France \\ and \\ Department of Engineering Physics, Chalmers University of Technology, \\ S-41296 Gothenburg, Sweden
}

Received 6 February 2006

\begin{abstract}
We analyze optical parametric interactions in nonlinear magneto-optical media simultaneously possessing a second-order nonlinearity and a spatial modulation of its linear optical properties. An analytic solution for the conversion efficiency of energy transfer from an optical pump to a signal wave is presented, and we discuss the possibility of tunability that is added to the parametric process.
\end{abstract}

Keywords: Optical parametric processes; magneto-optical interactions; phase-matching techniques.

\section{Introduction}

Three-wave coherent interactions of the class $\omega_{3} \rightarrow \omega_{1}+\omega_{2}$ with all energy exchanges occurring between the three field components without loss to the material medium, have been almost exclusively analyzed with linearly polarized light fields, in linearly birefringent (anisotropic) crystals where phase matching can be achieved by exploiting the linear birefringence. An alternative approach is to exploit the circular birefringence, natural or artificially induced, to achieve phase matching. This has already been discussed in the case of second and third harmonic generation in the case of optically active crystals $^{1,2}$ (natural optical activity) for linearly polarized light, and has further been analyzed in artificially gyrotropic (magnetooptic) media, ${ }^{3-5}$ in which selection rules for circularly polarized field components have been derived, relating to the spin selection rules of single-photon parametric processes. The interest of such configurations is ultimately conditioned by the 
possibility of compensating the dispersion mismatch with the circular birefringence, induced through the Faraday effect.

Meanwhile, since the early days of nonlinear optics, a parallel track has been the proposition to employ Bragg gratings as the means for achieving phase matching in nonlinear processes, as earlier suggested by Bloembergen and Sievers ${ }^{6}$ for parametric down-conversion and Brillouin scattering, employing periodic laminar structures. For the special case of second-harmonic generation, Tang and Bey ${ }^{7}$ investigated the possibility of employing sinusoidal refractive index gratings, long before the invention of the today commonly employed fiber Bragg grating. A more generalized analysis of phase-matching of three-wave interactions in third-order nonlinear processes in gratings was performed by Yariv and Yeh. ${ }^{8}$ Phase matching of second harmonic generation using gratings with a phase discontinuity for light localization has also been analyzed. ${ }^{9}$

Recently, the use of nonlinear fiber Bragg gratings for the simultaneous compression and amplification of optical pulses has also been suggested, ${ }^{10}$ in a technique where cross-phase modulation in the presence of optical Kerr effect together with the dispersion introduced by the grating causes parametric amplification of weak optical signal pulses, meanwhile automatically achieving an efficient phase matching scheme.

In all these cases of spatially modulated media, exclusively all-optical interactions have been taken into account in the phase matching schemes, and it is the aim of the present work to introduce the features of tunability and polarization state control added by also including magneto-optical interactions.

The starting point of the analysis is the vectorial electromagnetic wave equation as derived from Maxwell's equations ${ }^{11}$

$$
\nabla \times \nabla \times \mathbf{E}(\mathbf{r}, t)+\frac{1}{c^{2}} \frac{\partial^{2} \mathbf{E}(\mathbf{r}, t)}{\partial t^{2}}=-\mu_{0} \frac{\partial^{2} \mathbf{P}(\mathbf{r}, t)}{\partial t^{2}},
$$

in which $\mathbf{E}(\mathbf{r}, t)$ and $\mathbf{P}(\mathbf{r}, t)$ are the real-valued electromagnetic field and polarization density vectors, respectively. Throughout the analysis of three-wave interactions carried out in the present work, we for the complex-valued envelopes of the electric field adopt the convention

$$
\mathbf{E}(\mathbf{r}, t)=\sum_{m=1}^{3} \operatorname{Re}\left[\mathbf{E}_{\omega_{m}} \exp \left(-i \omega_{m} t\right)\right],
$$

where $\omega_{1}, \omega_{2}$, and $\omega_{3}$, denote the angular frequencies of the signal, idler, and pump waves, respectively, and similarly for the envelope of the electric polarization density

$$
\mathbf{P}(\mathbf{r}, t)=\sum_{m=1}^{3} \operatorname{Re}\left[\mathbf{P}_{\omega_{m}} \exp \left(-i \omega_{m} t\right)\right],
$$

the latter having its field envelope comprising the linear optical part

$$
\mathbf{P}_{\omega_{m}}^{(\mathrm{L})}=\varepsilon_{0}\left(\chi^{\mathrm{ee}} \mathbf{E}_{\omega_{m}}+\chi^{\mathrm{eem}} \mathbf{E}_{\omega_{m}} \mathbf{B}_{0}\right)
$$


and a nonlinear part

$$
\mathbf{P}_{\omega_{m}}^{(\mathrm{NL})}=\varepsilon_{0}\left(\chi^{\mathrm{eee}} \mathbf{E}_{\omega_{j}} \mathbf{E}_{\omega_{k}}+\chi^{\mathrm{eeem}} \mathbf{E}_{\omega_{j}} \mathbf{E}_{\omega_{k}} \mathbf{B}_{0}\right),
$$

where the field product $\mathbf{E}_{\omega_{j}} \mathbf{E}_{\omega_{k}}$ refers to the particular combination of two electrical field components giving rise to a a field at angular frequency $\omega_{m}=\omega_{j}+\omega_{k}$, taking into account the property $\mathbf{E}_{\omega_{j}}^{*}=\mathbf{E}_{-\omega_{j}}$ of conjugated fields.

In the linear part of the constitutive relation, $\chi^{\text {ee }}$ is the linear optical susceptibility tensor of the medium and $\chi^{\text {eem }}$ the linear magneto-optical susceptibility. In isotropic media these tensors in Eq. (2) evaluate to the well-known linear source terms $\chi_{x x}^{\mathrm{ee}} \mathbf{E}_{\omega_{m}}$ and $\chi_{x y z}^{\mathrm{eem}} \mathbf{E}_{\omega_{m}} \times \mathbf{B}_{0}$ respectively, the latter governing the artificially induced gyrotropy (Faraday effect). Similarly, in the nonlinear part of the constitutive relation, $\chi^{\text {eee }}$ is the nonlinear, second-order optical susceptibility tensor of the medium and $\chi^{\text {eeem }}$ its magneto-optical modification in the presence of static magnetic field.

In grating-assisted parametric interactions such as those considered here, the linear optical and magneto-optical properties are spatially modulated, creating an effective stop band in the spectrum. At the edges of this stop band, the group velocity dispersion as experienced by the traveling light waves, or equivalently their energy velocity dispersion, ${ }^{12,13}$ is considerably enhanced, and by employing this artificially introduced dispersion for any of the frequency components of the threewave interaction, an effective phase-matching scheme can be obtained also in media which otherwise are poorly fit for the regular phase matching techniques based on material dispersion and anisotropies.

The present work is divided into sections as follows: In Sec. 2, the fundamental linear and nonlinear optical properties of the magneto-optical medium is analyzed, with the constitutive relations in Sec. 3 applied to the analysis of parametric processes and wave propagation in a waveguiding structure. In Sec. 4, the linear band structure and dispersion characteristics of the magneto-optical grating is analyzed from the basis of the Bloch theory. These characteristics are essential for the further understanding of the phase matching requirements and tunability issues as discussed later on in Secs. 5 and 6, in which the wave equation is solved under the non-depleted pump approximation, providing an analytic solution to the conversion efficiency of the parametric process. In Sec. 7 the obtained results are discussed and illustrated with graphs of the signal amplification as a function of the applied static magnetic field for several configurations, with the conclusions summarized in Sec. 8.

\section{The Medium of Interaction}

For the present analysis we will assume a medium which is isotropic in its linear constitutive relations, meanwhile possessing a second-order nonlinearity supporting optical parametric interactions. The candidates for such media must, out of necessity, belong to any of the cubic point-symmetry groups $432, \overline{4} 3 \mathrm{~m}$, or $23 .{ }^{14}$ 
The remaining cubic point-symmetry groups $\mathrm{m} 3$ and $\mathrm{m} 3 \mathrm{~m}$ both possess inversion symmetry and do hence prohibit any second-order optical parametric interactions. For the sake of simplicity, we choose for the following analysis a medium belonging to point-symmetry group $\overline{4} 3 \mathrm{~m}$, or zinc-blende structure, in which the linear optical and magneto-optical properties are isotropic, and in order to further simplify the analysis we also choose a geometry in which the wave propagation occur in the $\langle 111\rangle$-direction of the crystalline lattice, this direction taken as the $z$-axis of the Cartesian coordinate system of the laboratory reference frame.

The elements of the susceptibility tensors evaluated in the laboratory reference frame $(x, y, z)$ are for the rank-three all-optical tensor $\chi^{\text {eee }}$ obtained in terms of the elements in the crystal frame $(X, Y, Z)$ from the transformation rule for polar tensors

$$
\chi_{i j k}^{\mathrm{eee}}=R_{i I} R_{j J} R_{k K} \chi_{I J K}^{\mathrm{eee}},
$$

and similarly for the rank-four magneto-optical tensor $\chi^{\text {eeem }}$ from the transformation rule for axial tensors

$$
\chi_{i j k l}^{\mathrm{eeem}}=\operatorname{det}(\mathbf{R}) R_{i I} R_{j J} R_{k K} R_{l L} \chi_{I J K L}^{\mathrm{eeem}},
$$

where $\mathbf{R}$ is the rotational matrix composed of the direction cosines between the Cartesian coordinate axes of the crystal and laboratory reference frames. The elements of the second-order susceptibility tensors expressed in the laboratory reference frame are listed in Tables 1 and 2. For this choice of orientation of the $z$-axis of the laboratory frame, the linear and nonlinear optical properties of the medium are all invariant under rotation of the medium around the axis of wave propagation, hence eliminating any nonlinearly induced birefringence or cross-phase modulation between linearly polarized field components.

One well-known magneto-optical material belonging to the zinc-blende pointsymmetry group $\overline{4} 3 \mathrm{~m}$ is the semi-magnetic semiconductor $\mathrm{Cd}_{1-x} \mathrm{Mn}_{x} \mathrm{Te}$, which has been proven to also possess high optical nonlinearities. ${ }^{15,16}$ The chosen configuration also has the advantage that it connects to the earlier suggestions of using composite

Table 1. Nonzero elements of the second-order optical susceptibility tensor $\chi^{\text {eee }}$ for point symmetry group $\overline{4} 3 \mathrm{~m}$, in the laboratory coordinate frame $(x, y, z)$, with the $z$ axis in the direction of the crystal $\langle 111\rangle$-direction. Capital indices of tensor elements relate to the Cartesian coordinate axes intrinsic of the crystal reference frame.

\begin{tabular}{l} 
Nonzero elements $^{\mathrm{a}}$ of $\chi_{i j k}^{\mathrm{eee}}$ \\
\hline$\chi_{x x x}^{\mathrm{eee}}=\chi_{y y y}^{\mathrm{eee}}=\chi_{z z z}^{\mathrm{eee}} / 2=a$ \\
$\chi_{x x y}^{\mathrm{eee}}=\chi_{y y x}^{\mathrm{eee}}=\chi_{x y x}^{\mathrm{eee}}=\chi_{y x y}^{\mathrm{eee}}=\chi_{x y y}^{\mathrm{eee}}=\chi_{y x x}^{\mathrm{eee}}=-a$ \\
$\chi_{x x z}^{\mathrm{ee}}=\chi_{z x x}^{\mathrm{eee}}=\chi_{y y z}^{\mathrm{eee}}=\chi_{z y y}^{\mathrm{eee}}=\chi_{x z x}^{\mathrm{eee}}=\chi_{y z y}^{\mathrm{eee}}=-a$ \\
$a \equiv \chi_{X Y Z}^{\mathrm{eee}} / 3^{1 / 2}$
\end{tabular}

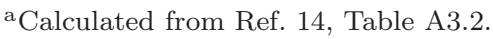


Table 2. Nonzero elements of the third-order magneto-optical susceptibility tensor $\chi^{\text {eeem }}$ for point symmetry group $\overline{4} 3 \mathrm{~m}$, in the laboratory coordinate frame $(x, y, z)$, with the $z$ axis in the direction of the crystal $\langle 111\rangle$-direction. Capital indices of tensor elements relate to the Cartesian coordinate axes intrinsic of the crystal reference frame.

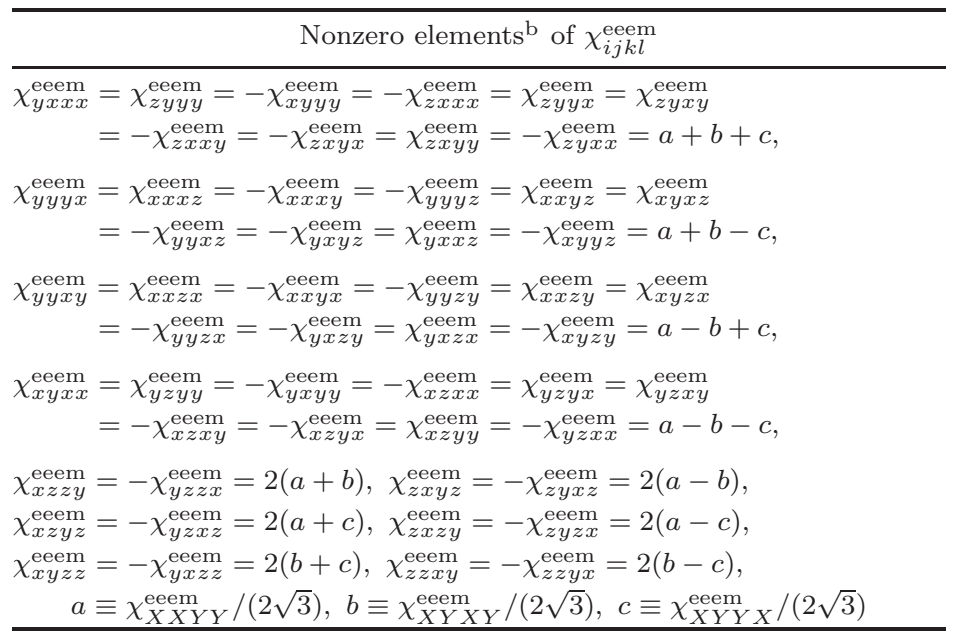

${ }^{\mathrm{b}}$ Calculated from Ref. 18, Table II.

layered structures of $\mathrm{GaP}$ and $\mathrm{GaAs}$ for the phase matching, in a geometry in which the layers were grown epitaxially with their $\langle 111\rangle$-axes normal to the layer surfaces. ${ }^{6}$

To support an all-optical second-order nonlinearity, or equivalently a secondorder electric dipolar contribution to the electric polarization density, the medium must as a basic requirement not possess inversion symmetry. ${ }^{17}$ This follows from the transformation rule (4) of polar tensors, which if the medium under investigation is invariant under the inversion transformation $R_{i I}=-\delta_{i I}$ gives the well-known identity $\chi_{i j k}^{\mathrm{eee}}=0$. This result is easily generalized to yield the fact that if the inversion operation is a symmetry operation of the medium, then all tensor elements of any all-optical source term of the polarization density which involves an even number of electric field components must identically vanish.

From this fact, one could easily be misled to believe that the magnetooptical nonlinear susceptibility $\chi_{i j k l}^{\text {eeem }}$ could support a second-order nonlinearity, as those source terms involve the products of three fields, two electric and one magnetic one. However, from the transformation rule (5) of axial tensors, one directly finds that invariance of the second-order magneto-optical properties under any inversion operation with $\operatorname{det}(\mathbf{R})=-1$ still requires the tensor elements $\chi_{i j k l}^{\text {eeem }}$ to be identically zero. This result is, similar to the all-optical one, easily generalized to yield the fact that if the inversion operation is a symmetry operation of the medium, then all tensor elements of any magnetooptical source term of the polarization density which involves an even number 
of electric fields and an odd number of magnetic fields must identically vanish as well.

It should be emphasized here that we in the present terminology use the term isotropic to denote a material property which is invariant under any proper rotation, with $\operatorname{det}(\mathbf{R})=1$, which does not automatically include inversion symmetry as an intrinsic property. For example, the chosen point-symmetry group $\overline{4} 3 \mathrm{~m}$ is isotropic in its linear optical properties, being a non-birefringent medium in the absence of static magnetic fields, while it still does not possess inversion symmetry, hence still possessing a second-order optical nonlinearity.

\section{Wave Propagation}

In many cases the nonlinearity and its associated inter-frequency wave interaction can be comparatively weak, and so it becomes important to keep the optical wave confined to the active region so as to ensure that the optical intensity is kept at a sufficiently high level. One solution in this respect is to employ wave-guiding structures while also maintaining a spatial modulation of the optical properties of the medium in the direction of wave propagation, such as for example in fiber Bragg gratings. ${ }^{19}$ One advantage with this solution is also that it provides a means to keep the transverse spatial overlap of the waves in the interaction fixed, thus considerably simplifying the setup in applications. For the sake of self-consistency of the following nonlinear analysis, the separation of the radial and longitudinal dependence of the optical waves will now be outlined.

By expanding the spatial differentiation as appearing in the general wave propagation described by Eq. (1), and by furthermore assuming that the spatial variation of the optical properties of the medium is weak enough to allow the assumption

$$
\nabla\left(\nabla \cdot \mathbf{E}_{\omega_{m}}\right) \ll \nabla^{2} \mathbf{E}_{\omega_{m}}
$$

the wave equation governing the spatial evolution of the complex-valued electric field components becomes

$$
\nabla^{2} \mathbf{E}_{\omega_{m}}+\frac{\omega_{m}^{2}}{c^{2}}\left[\boldsymbol{\delta}+\chi^{\mathrm{ee}}+\chi^{\mathrm{eem}} \mathbf{B}_{0}\right] \mathbf{E}_{\omega_{m}}=-\mu_{0} \omega_{m}^{2} \mathbf{P}_{\omega_{m}}^{(\mathrm{NL})},
$$

where, in the Cartesian coordinate system of the laboratory reference frame, $\delta \equiv$ $\mathbf{e}_{i} \mathbf{e}_{j} \delta_{i j}$ is the rank-two unitary tensor, $\chi^{\mathrm{ee}} \equiv \mathbf{e}_{i} \mathbf{e}_{j} \chi_{i j}^{\mathrm{ee}}\left(\mathbf{r}, \omega_{m}\right)$ is the rank-two tensor governing the linear optical polarization density and $\chi^{\mathrm{eem}} \equiv \mathbf{e}_{i} \mathbf{e}_{j} \mathbf{e}_{k} \chi_{i j k}^{\mathrm{eem}}\left(\mathbf{r}, \omega_{m}\right)$ is the rank-three tensor governing the linear magneto-optical polarization density, ${ }^{20}$ the latter two being spatially dependent quantities to be evaluated at respective angular frequency $\omega_{m}$. For the present case of a medium having its linear optical properties isotropic, these tensors take the simple forms $\chi_{i j}^{\mathrm{ee}}=\delta_{i j} \chi_{x x}^{\mathrm{ee}}$ and $\chi_{i j k}^{\mathrm{eem}}=$ $\epsilon_{i j k} \chi_{x y z}^{\mathrm{eem}}$, with $\delta_{i j}$ and $\epsilon_{i j k}$ being the Kronecker delta and Levi-Civitá permutation tensors ${ }^{21}$ respectively. 
The direction of the externally applied magnetic field is taken as collinear with the direction of wave propagation, with $\mathbf{B}_{0}=B_{0}^{z} \mathbf{e}_{z}$ in the Faraday configuration. ${ }^{22}$ The medium in which the wave propagation takes place is at angular frequency $\omega_{m}$ possessing a linear refractive index $n_{m}^{2}(x, y, z)=1+\chi_{x x}^{\mathrm{ee}}\left(-\omega_{m} ; \omega_{m}\right)$ and magnetooptical gyration coefficient $\gamma_{m}(x, y, z)=i \chi_{x y z}^{\text {eem }}\left(-\omega_{m} ; \omega_{m}, 0\right) B_{0}^{z}$ spatially modulated as

$$
\begin{aligned}
& \frac{n_{m}^{2}(x, y, z)-\tilde{n}_{m}^{2}}{\tilde{n}_{m}}=a_{m} \cos (K z+\vartheta)+a_{m}^{\prime} f(x, y), \\
& \frac{\gamma_{m}(x, y, z)-\tilde{\gamma}_{m}}{\tilde{n}_{m}}=b_{m} \cos (K z+\vartheta)+b_{m}^{\prime} f(x, y),
\end{aligned}
$$

where $\tilde{n}_{m}$ and $\tilde{\gamma}_{m}$ are the respective bias indices and gyration coefficients, as inherent of the bulk medium well outside the waveguiding core, and where $K$ is the magnitude of the grating vector, related to the grating period $\Lambda$ as $K=2 \pi / \Lambda$. Although intentionally introduced defects or other perturbations in magneto-optical gratings have been shown to provide a strong polarization state selectivity of the light localization, in a linear ${ }^{23}$ as well as nonlinear ${ }^{24}$ optical regime, we for the present case focus attention on a grating with homogeneously modulated phase, with $\vartheta$ being a real-valued constant. The transverse real-valued distribution function $f(x, y)$, describing the waveguiding cross-section profile, is taken to have its maximum value of $f(0,0)=1$ at the centre of the core, outside of which it rapidly drops towards zero. For simplicity, the nonlinear optical and nonlinear magneto-optical properties at the respective angular frequencies are assumed to be homogeneous and non-modulated.

Each frequency component of the complex envelope of the electric field is expressed in the circularly polarized basis $\mathbf{e}_{ \pm}=\left(\mathbf{e}_{x} \pm i \mathbf{e}_{y}\right) / 2^{1 / 2}$ and separated into a factorized form as

$$
\begin{aligned}
\mathbf{E}_{\omega_{m}}= & \mathbf{e}_{+} F_{m}^{+}(x, y) A_{m}^{\mathrm{f}+}(z) \exp \left(i \beta_{m}^{+} z\right)+\mathbf{e}_{-} F_{m}^{-}(x, y) A_{m}^{\mathrm{f}-}(z) \exp \left(i \beta_{m}^{-} z\right) \\
& +\mathbf{e}_{+}^{*} F_{m}^{-}(x, y) A_{m}^{\mathrm{b}+}(z) \exp \left(-i \beta_{m}^{-} z\right)+\mathbf{e}_{-}^{*} F_{m}^{+}(x, y) A_{m}^{\mathrm{b}-}(z) \exp \left(-i \beta_{m}^{+} z\right),
\end{aligned}
$$

where $\mathrm{a} \pm$ in the superscripts of the forward or backward traveling field envelopes $A_{m}^{\mathrm{f} \pm}$ or $A_{m}^{\mathrm{b} \pm}$ denotes left/right circular polarization state, taking into account also the direction of propagation. In Eq. (8), $\beta_{m}^{ \pm}$are the respective linear propagation coefficients of the waveguided modes, to be determined later on from the transverse profile of the waveguide by the modal eigenvalue equation. In this separation, the envelopes $A_{m}^{\mathrm{f} \pm}$ and $A_{m}^{\mathrm{b} \pm}$ have the physical dimension of the electric field, or $\mathrm{V} / \mathrm{m}$ in SI units, while the transverse mode profiles $F_{m}^{ \pm}(x, y)$ are dimensionless and normalized to yield

$$
\iint F_{m}^{+}(x, y) d x d y=\iint F_{m}^{-}(x, y) d x d y=1,
$$


with the integration to be performed over the entire transverse extent of the modes. The form (8) of the separation of the transverse and longitudinal spatial dependence of the electromagnetic field, with identical mode profiles and propagation coefficients assumed for counter-propagating components of opposite circular polarization state, is justified by the observation that the backward traveling components experience an effective change of sign of the applied magnetic field, hence switching roles of the circular polarization states, as compared to the forward traveling components. In this form, the variable separation in Eq. (8) assumes a constant phase in the transverse $x y$-plane at all longitudinal positions; this hence excludes the possibility of analysis of radiation modes, limiting the discussion to the wave-guided modes.

As the factorized form (8) of the electromagnetic field is inserted into the wave equation (6), furthermore using the linear optical properties of the medium as in Eqs. (7) and applying the slowly varying envelope approximation ${ }^{17}$ for the envelopes in the forward and backward directions of propagation, one obtains a coupled system of differential equations for the forward and backward traveling components. This system is of considerable algebraic complexity, and in order to simplify the following analysis we now apply the assumption that the pump $\left(\omega_{3}\right)$ and idler $\left(\omega_{2}\right)$ beams are out of Bragg resonance with the grating, as schematically illustrated in Fig. 1. Under this assumption, the backward traveling components of the pump and idler wave both become zero, and the simplified coupled system for the field envelopes becomes

$$
\begin{aligned}
& A_{1}^{\mathrm{f} \pm} \nabla_{\mathrm{T}}^{2} F_{1}^{ \pm}+F_{1}^{ \pm}\left[2 i \beta_{1}^{ \pm} \frac{\partial}{\partial z}+\left(\omega_{1} / c\right)^{2}\left(\tilde{n}_{1}^{2} \pm \tilde{\gamma}_{1}\right)-\beta_{1}^{ \pm 2}\right] A_{1}^{\mathrm{f} \pm} \\
& \quad+\left(\omega_{1} / c\right)^{2}\left(\tilde{n}_{1} / 2\right)\left(a_{1} \pm b_{1}\right) \exp \left(-2 i \alpha_{ \pm} z+i \vartheta\right) F_{1}^{ \pm} A_{1}^{\mathrm{b} \mp} \\
& \quad+\left(\omega_{1} / c\right)^{2} \tilde{n}_{1}\left(a_{1}^{\prime} \pm b_{1}^{\prime}\right) f(x, y) F_{1}^{ \pm} A_{1}^{\mathrm{f} \pm} \\
& \quad+2^{1 / 2}(1 \pm i)\left(\omega_{1} / c\right)^{2}\left(\chi_{x x x}^{\mathrm{eee}} \mp i \chi_{x x x z}^{\mathrm{eeem}} B_{0}^{z}\right) F_{3}^{\mp} F_{2}^{ \pm} A_{3}^{\mathrm{f} \mp} A_{2}^{\mathrm{f} \pm *} \exp \left(i \beta_{ \pm} z\right)=0
\end{aligned}
$$

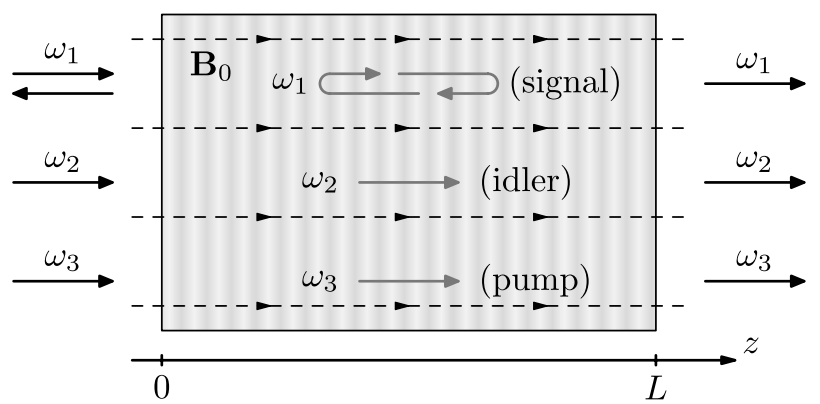

Fig. 1. Schematic figure of the setup of the singly resonant parametric process in a nonlinear magneto-optical Bragg grating in the Faraday configuration, in which the static magnetic field $\mathbf{B}_{0}$ is directed along the axis of wave propagation. Of the three interacting waves, only the signal is close to resonance of the Bragg grating, causing the parametric interaction to occur only in the forward direction of propagation. 


$$
\begin{aligned}
& A_{1}^{\mathrm{b} \mp} \nabla_{\mathrm{T}}^{2} F_{1}^{ \pm}-F_{1}^{ \pm}\left[2 i \beta_{1}^{ \pm} \frac{\partial}{\partial z}-\left(\omega_{1} / c\right)^{2}\left(\tilde{n}_{1}^{2} \pm \tilde{\gamma}_{1}\right)+\beta_{1}^{ \pm 2}\right] A_{1}^{\mathrm{b} \mp} \\
& \quad+\left(\omega_{1} / c\right)^{2}\left(\tilde{n}_{1} / 2\right)\left(a_{1} \pm b_{1}\right) \exp \left(2 i \alpha_{ \pm} z-i \vartheta\right) F_{1}^{ \pm} A_{1}^{\mathrm{f} \pm} \\
& \quad+\left(\omega_{1} / c\right)^{2} \tilde{n}_{1}\left(a_{1}^{\prime} \pm b_{1}^{\prime}\right) f(x, y) F_{1}^{ \pm} A_{1}^{\mathrm{b} \mp}=0 \\
& A_{2}^{\mathrm{f} \pm} \nabla_{\mathrm{T}}^{2} F_{2}^{ \pm}+F_{2}^{ \pm}\left[2 i \beta_{2}^{ \pm} \frac{\partial}{\partial z}+\left(\omega_{2} / c\right)^{2}\left(\tilde{n}_{2}^{2} \pm \tilde{\gamma}_{2}\right)-\beta_{2}^{ \pm 2}\right] A_{2}^{\mathrm{f} \pm} \\
& \quad+\left(\omega_{2} / c\right)^{2} \tilde{n}_{2}\left(a_{2}^{\prime} \pm b_{2}^{\prime}\right) f(x, y) F_{2}^{ \pm} A_{2}^{\mathrm{f} \pm} \\
& \quad+2^{1 / 2}(1 \pm i)\left(\omega_{2} / c\right)^{2}\left(\chi_{x x x}^{\mathrm{eee}} i \chi_{x x x z}^{\mathrm{eeem}} B_{0}^{z}\right) F_{3}^{\mp} F_{1}^{ \pm} A_{3}^{\mathrm{f} \mp} A_{1}^{\mathrm{f} \pm *} \exp \left(i \beta_{\mp} z\right)=0 \\
& A_{3}^{\mathrm{f} \pm} \nabla_{\mathrm{T}}^{2} F_{3}^{ \pm}+F_{3}^{ \pm}\left[2 i \beta_{3}^{ \pm} \frac{\partial}{\partial z}+\left(\omega_{3} / c\right)^{2}\left(\tilde{n}_{3}^{2} \pm \tilde{\gamma}_{3}\right)-\beta_{3}^{ \pm 2}\right] A_{3}^{\mathrm{f} \pm} \\
& \quad+\left(\omega_{3} / c\right)^{2} \tilde{n}_{3}\left(a_{3}^{\prime} \pm b_{3}^{\prime}\right) f(x, y) F_{3}^{ \pm} A_{3}^{\mathrm{f} \pm} \\
& \quad+2^{1 / 2}(1 \pm i)\left(\omega_{3} / c\right)^{2}\left(\chi_{x x x}^{\mathrm{eee}} \mp i \chi_{x x x z}^{\mathrm{eeem}} B_{0}^{z}\right) F_{1}^{\mp} F_{2}^{\mp} A_{1}^{\mathrm{f} \mp} A_{2}^{\mathrm{f} \mp} \exp \left(-i \beta_{\mp} z\right)=0
\end{aligned}
$$

in which $\nabla_{\mathrm{T}}=(\partial / \partial x, \partial / \partial y)$ is the transverse part of the nabla operator, $\tilde{n}_{m}^{2} \pm \tilde{\gamma}_{m}$ are the squared effective bias bulk refractive indices for the left/right circularly polarized modes,

$$
\alpha_{ \pm}=\beta_{1}^{ \pm}-K / 2
$$

are the left/right circularly polarized signal wave vector detunings from the Bragg resonance in reflection, and

$$
\beta_{ \pm}=\beta_{3}^{\mp}-\beta_{2}^{ \pm}-\beta_{1}^{ \pm}
$$

are the corresponding phase mismatch parameters between the idler, signal and pump beams. In Eqs. (9) it is to be implicitly understood that the nonlinear optical and magneto-optical susceptibilities $\chi_{x x x}^{\mathrm{eee}}$ and $\chi_{x x x z}^{\mathrm{eeem}}$ are to be evaluated according to the context of the frequencies of the field envelopes, counting the angular frequencies of complex conjugated fields as negative.

The system (9) is generally not separable into its transverse and longitudinally dependent parts, due to their coupling in the presence of nonlinear terms. However, by neglecting the nonlinear optical modification to the transverse mode profile, hence relying on that the profile to a good approximation can be determined from the linear optical properties of the waveguide in the transverse plane $(x, y)$, the system separates into a transverse part

$$
\left[\nabla_{\mathrm{T}}^{2}+\frac{\omega_{m}^{2}}{c^{2}}\left(\tilde{n}_{m}^{2} \pm \tilde{\gamma}_{m}+\tilde{n}_{m}\left(a_{m}^{\prime} \pm b_{m}^{\prime}\right) f(x, y)\right)-\beta_{m}^{ \pm 2}\right] F_{m}^{ \pm}=0,
$$

for $m=1,2,3$, and a longitudinal part

$$
\begin{aligned}
& \frac{\partial A_{1}^{\mathrm{f} \pm}}{\partial z}-\kappa_{ \pm}^{*} A_{1}^{\mathrm{b} \mp} \exp \left(-2 i \alpha_{ \pm} z\right)-i C_{1}^{ \pm} A_{3}^{\mathrm{f} \mp} A_{2}^{\mathrm{f} \pm *} \exp \left(i \beta_{ \pm} z\right)=0 \\
& \frac{\partial A_{1}^{\mathrm{b} \mp}}{\partial z}-\kappa_{ \pm} A_{1}^{\mathrm{f} \pm} \exp \left(2 i \alpha_{ \pm} z\right)=0
\end{aligned}
$$




$$
\begin{aligned}
& \frac{\partial A_{2}^{\mathrm{f} \pm}}{\partial z}-i C_{2}^{ \pm} A_{3}^{\mathrm{f} \mp} A_{1}^{\mathrm{f} \pm *} \exp \left(i \beta_{ \pm} z\right)=0, \\
& \frac{\partial A_{3}^{\mathrm{f} \pm}}{\partial z}-i C_{3}^{ \pm} A_{1}^{\mathrm{f} \mp} A_{2}^{\mathrm{f} \mp} \exp \left(-i \beta_{ \pm} z\right)=0
\end{aligned}
$$

where the effective linear coupling coefficient experienced by the signal wave due to the periodic spatial modulation of the grating was defined as

$$
\kappa_{ \pm}=-i \frac{\omega_{1}^{2} \tilde{n}_{1}}{4 c^{2} \beta_{1}^{ \pm}}\left(a_{1} \pm b_{1}\right) \exp (-i \vartheta) .
$$

In the following, the subscripts of $a_{1}$ and $b_{1}$ are dropped as no ambiguity is present in their meaning for the singly resonant case. In Eqs. (13), the introduced nonlinear coefficients are defined as ${ }^{4}$

$$
\begin{aligned}
C_{1}^{ \pm} & =\frac{(1 \pm i) \omega_{1}^{2}}{2^{1 / 2} c^{2} \beta_{1}^{ \pm}}\left(\chi_{x x x}^{\mathrm{eee}} \mp i \chi_{x x x z}^{\mathrm{eeem}} B_{0}^{z}\right) \iint F_{3}^{\mp} F_{2}^{ \pm} d x d y, \\
C_{2}^{ \pm} & =\frac{(1 \pm i) \omega_{2}^{2}}{2^{1 / 2} c^{2} \beta_{2}^{ \pm}}\left(\chi_{x x x}^{\mathrm{eee}} \mp i \chi_{x x x z}^{\mathrm{eeem}} B_{0}^{z}\right) \iint F_{3}^{\mp} F_{1}^{ \pm} d x d y, \\
C_{3}^{ \pm} & =\frac{(1 \pm i) \omega_{3}^{2}}{2^{1 / 2} c^{2} \beta_{3}^{ \pm}}\left(\chi_{x x x}^{\mathrm{eee}} \mp i \chi_{x x x z}^{\mathrm{eeem}} B_{0}^{z}\right) \iint F_{1}^{\mp} F_{2}^{\mp} d x d y,
\end{aligned}
$$

in which the mode profiles as calculated from Eq. (12) were incorporated in terms of overlap integrals over the spatial profiles. In Eq. (14), the initial reference phase $\vartheta$ of the grating can be chosen arbitrarily, thus allowing for the linear coupling coefficients $\kappa_{ \pm}$to both simultaneously be chosen as real-valued quantities in loss-less media. The tensor elements $\chi_{x x x}^{\text {eee }}$ govern the all-optical, electric dipolar contribution to the parametric interaction, while $\chi_{x x x z}^{\text {eeem }}$ govern the small but non-negligible magneto-optically induced contribution.

Notice that in cases in which the active region of the core also defines the region of where the magneto-optical interaction takes place, that is to say if $b_{m}^{\prime} \neq 0$, the effective refractive index contrast between the core and cladding will be experiencing an opposite shift for orthogonal circular polarization states. This will lead to one circular polarization state being more strongly guided than the other, with their roles interchanged whenever the direction of the static magnetic field is switched. This possibility is, in the present analysis, included by allowing for the spatial mode profiles of the orthogonal circular polarization states to have different transverse distribution functions $F_{+}(x, y)$ and $F_{-}(x, y)$.

The separation of the transverse and longitudinal dependence of the optical wave essentially limits the validity of the present analysis to the level of the weak guidance approximation, ${ }^{25}$ which however still makes the model applicable to a large class of single-mode structures. Another benefit with this level of approximation is that we are in position to develop an analytical theory of the wave propagation, focusing on the nonlinear aspect of propagation rather than the waveguiding properties. For more complicated waveguiding geometries, or for large contrast in refractive indices between the core and surrounding cladding, usually numerical models for 
the wave propagation need to be employed. ${ }^{26}$ It is important here to note that in forming the system given by Eqs. (13), the non-resonant terms of the idler and pump are assumed to be of angular frequencies which do not coincide with higher order resonances of the grating, that is to say, their respective propagation coefficients $\beta_{2}^{ \pm}$and $\beta_{3}^{ \pm}$must not coincide with integer multiples of $\pi / \Lambda$.

Within the scope of validity of the approximations leading to the separated form of Eqs. (12) and (13) of the field envelopes, the propagation coefficients $\beta_{k}^{ \pm}$are fully determined by the two-dimensional partial differential equation (13). Naturally, the explicit form of the frequency dependence of these coefficients depend on the transverse distribution function $f(x, y)$, which in the present work is taken as a generic function, as the focus here is on the wave propagation characteristics. For a detailed analysis of the explicit forms of the propagation coefficients for various profiles $f(x, y)$, we refer to Refs. $25-27$.

\section{Linear Band Structure of Magneto-Optical Gratings}

At the heart of the optical parametric process described by Eqs. (13) lies the phase matching, which depends not only on any modifications of the wave propagation as imposed by the waveguiding structure, but also on the magneto-optical properties of the medium and in particular the introduction of non-reciprocity, ${ }^{22,28}$ which effectively distinguishes between parametric processes in the forward and backward directions relative to the applied static magnetic field.

In some cases, the change of the bulk properties by inclusion of the Faraday effect can be sufficient to provide an efficient phase matching scheme; ${ }^{3}$ however, whenever such schemes cannot fulfill the phase matching criterion, operation at the edge of the optical band gap of a periodic structure can be employed instead. ${ }^{6-8}$ The feature of also including a magneto-optical effect in these structures can be appreciated as one considers their linear behavior as a function of the applied magnetic field strength.

The band structure of magneto-optical gratings can in fact be discussed qualitatively in a linear optical domain without having to specify the exact longitudinal profile of the periodic medium, as will now be shown. This analysis serves to support some arguments and qualitative discussions on phase matching of nonlinear processes as will be discussed in Sec. 5. Later on, the frequency of the signal wave will be taken as close to that corresponding to resonance of the grating. However, in order to simplify the notation, the general and more qualitative analysis as considered here is carried out for an arbitrary frequency.

The general starting point for the band structure analysis is the one-dimensional time-dependent wave equation of linear magneto-optic media in the Faraday configuration, ${ }^{22}$

$$
\frac{\partial^{2} \mathbf{E}(z, t)}{\partial z^{2}}-\frac{1}{c^{2}} \frac{\partial^{2}}{\partial t^{2}}\left[n^{2}(z) \mathbf{E}(z, t)+\mathbf{g}(z) \times \frac{\partial \mathbf{E}(z, t)}{\partial t}\right]=\mathbf{0},
$$


in which the gyration vector $\mathbf{g}(z)$ for loss-less media is related to the linear magnetooptical susceptibility and the static magnetic field as $\mathbf{g}=-i\left(\chi_{x y z}^{\mathrm{eem}} / \omega\right) \mathbf{B}_{0}$.

For our current purposes, it is sufficient to consider a magneto-optic medium with its optical and magneto-optical parameters obeying the periodicity conditions

$$
n^{2}(z+\Lambda)=n^{2}(z), \quad \gamma(z+\Lambda)=\gamma(z),
$$

where as previously $n^{2}=1+\chi_{x x}^{\mathrm{ee}}$ is the square of the optical refractive index of the medium, $\gamma=i B_{0}^{z} \chi_{x y z}^{\mathrm{eem}}$ is the gyration coefficient, and $\Lambda$ the spatial period of their modulation. The periodicity of the material parameters implies that they can be expanded into Fourier series as

$$
\begin{aligned}
n^{2}(z) & =\sum_{m=-\infty}^{\infty} \tilde{n}_{m}^{2} \exp (i m K z), \\
\gamma(z) & =\sum_{m=-\infty}^{\infty} \tilde{\gamma}_{m} \exp (i m K z)
\end{aligned}
$$

in which $K=2 \pi / \Lambda$ is the magnitude of the reciprocal grating vector. In order to simplify the analysis, it is now assumed that the dominating terms of the Fourier expansions in Eqs. (17) are the first-order terms with $m=0$ and $m= \pm 1$, as

$$
\begin{aligned}
n^{2}(z) & \approx \tilde{n}_{0}^{2}+\tilde{n}_{-1}^{2} \exp (-i K z)+\tilde{n}_{1}^{2} \exp (i K z), \\
\gamma(z) & \approx \tilde{\gamma}_{0}+\tilde{\gamma}_{-1} \exp (-i K z)+\tilde{\gamma}_{1} \exp (i K z) .
\end{aligned}
$$

This is in particular a highly valid approximation for fiber Bragg gratings or gratings inscribed in other waveguiding structures, which to a high level of accuracy can be modeled as being sinusoidal in their spatial modulation of the refractive index distribution. $^{19}$

The transverse and circularly polarized eigenmodes of the electromagnetic field in a one-dimensional magneto-photonic crystal, which in order to be relevant for band structure analysis has to be assumed to be of infinite longitudinal extent, are characterized by wave numbers $k_{ \pm}$as

$$
\mathbf{E}(\mathbf{r}, t)=\operatorname{Re}\left[\mathbf{e}_{+} E_{k_{+}}(z, t)+\mathbf{e}_{-} E_{k_{-}}(z, t)\right],
$$

in which the complex-valued circularly polarized electric field components are

$$
E_{k_{ \pm}}(z, t)=u_{k_{ \pm}}(z) \exp \left[i\left(k_{ \pm} z-\omega_{ \pm} t\right)\right]
$$

with their envelopes satisfying the condition of periodicity

$$
u_{k_{ \pm}}(z+\Lambda)=u_{k_{ \pm}}(z) \text {. }
$$

From the Bloch theory ${ }^{29,30}$ the periodicity condition in Eq. (21) also implies that the electric field can be expressed in a Fourier expansion, as

$$
E_{k_{ \pm}}(z, t)=\sum_{m=-\infty}^{\infty} \tilde{E}_{m}^{ \pm} \exp \left[i\left(k_{ \pm}+m K\right) z-i \omega_{ \pm} t\right],
$$

in which $\tilde{E}_{m}^{ \pm}$are the Fourier coefficients of the circularly polarized eigenmodes. By inserting the Fourier series given by Eq. (22) and the approximate expression for the 
material parameters as given by Eqs. (18) into Eq. (15), one obtains, after some straightforward algebra and by projecting out phase matched terms, an infinite chain of equations for the Fourier coefficients of the electromagnetic field as

$$
\begin{aligned}
& \frac{\omega_{+}^{2}}{c^{2}}\left[\left(\tilde{n}_{+1}^{2}+\tilde{\gamma}_{+1}\right) \tilde{E}_{m-1}^{+}+\left(\tilde{n}_{-1}^{2}+\tilde{\gamma}_{-1}\right) \tilde{E}_{m+1}^{+}\right] \\
& +\left[\frac{\omega_{+}^{2}}{c^{2}}\left(\tilde{n}_{0}^{2}+\tilde{\gamma}_{0}\right)-\left(k_{+}+\frac{2 \pi m}{\Lambda}\right)^{2}\right] \tilde{E}_{m}^{+}=0, \\
& \frac{\omega_{-}^{2}}{c^{2}}\left[\left(\tilde{n}_{+1}^{2}-\tilde{\gamma}_{+1}\right) \tilde{E}_{m-1}^{-}+\left(\tilde{n}_{-1}^{2}-\tilde{\gamma}_{-1}\right) \tilde{E}_{m+1}^{-}\right] \\
& +\left[\frac{\omega_{-}^{2}}{c^{2}}\left(\tilde{n}_{0}^{2}-\tilde{\gamma}_{0}\right)-\left(k_{-}+\frac{2 \pi m}{\Lambda}\right)^{2}\right] \tilde{E}_{m}^{-}=0 .
\end{aligned}
$$

The goal now is to extract the dispersion relation $\omega(k)$ from these relations. By limiting to a fixed number terms in the solution chain given by Eqs. (23), say in total $2 M+1$ terms with $-M \leq m \leq M$, the eigenvalue problems for the normalized eigenfrequencies $\omega_{ \pm} \Lambda / \pi c$ as a function of normalized wave-vectors $k_{ \pm} \Lambda / \pi$ can be formulated as two tri-diagonal linear systems, one for each circular polarization state,

$$
\left(\begin{array}{ccccccc}
d_{-M}^{ \pm} & s_{-1}^{ \pm} & & & & & \\
s_{+1}^{ \pm} & d_{-M+1}^{ \pm} & s_{-1}^{ \pm} & & & & \\
& \ddots & \ddots & \ddots & & & \\
& & s_{+1}^{ \pm} & d_{0}^{ \pm} & s_{-1}^{ \pm} & & \\
& & & \ddots & \ddots & \ddots & \\
& & & & s_{+1}^{ \pm} & d_{M-1}^{ \pm} & s_{-1}^{ \pm} \\
& & & & & s_{+1}^{ \pm} & d_{M}^{ \pm}
\end{array}\right)\left(\begin{array}{c}
\tilde{E}_{-M}^{ \pm} \\
\vdots \\
\tilde{E}_{-1}^{ \pm} \\
\tilde{E}_{0}^{ \pm} \\
\tilde{E}_{1}^{ \pm} \\
\vdots \\
\tilde{E}_{M}^{ \pm}
\end{array}\right)=\mathbf{0}
$$

in which the diagonal elements are

$$
d_{m}^{ \pm}=\left(\tilde{n}_{0}^{2} \pm \tilde{\gamma}_{0}\right)\left(\frac{\omega_{ \pm} \Lambda}{\pi c}\right)^{2}-\left(\frac{k_{ \pm} \Lambda}{\pi}+2 m\right)^{2}
$$

for $m=-M, \ldots, M$, and with all sub- and super-diagonal elements being equal for each polarization state,

$$
s_{+1}^{ \pm}=\left(\tilde{n}_{+1}^{2} \pm \tilde{\gamma}_{+1}\right)\left(\frac{\omega_{ \pm} \Lambda}{\pi c}\right)^{2}, \quad s_{-1}^{ \pm}=\left(\tilde{n}_{-1}^{2} \pm \tilde{\gamma}_{-1}\right)\left(\frac{\omega_{ \pm} \Lambda}{\pi c}\right)^{2} .
$$

By requiring the determinant of the system matrix of the eigenvalue equation (24) to be zero, one obtains two $(2 M+1)$-order polynomial equations for the squared normalized eigenfrequencies as a function of the normalized wave vectors, with one polynomial for each circular polarization state of light. These equations form the implicit solution curves for the dispersion relations $\omega_{+}\left(k_{+}\right)$and $\omega_{-}\left(k_{-}\right)$, and their solution for the case of the left circular polarization state is shown in Fig. 2, for 


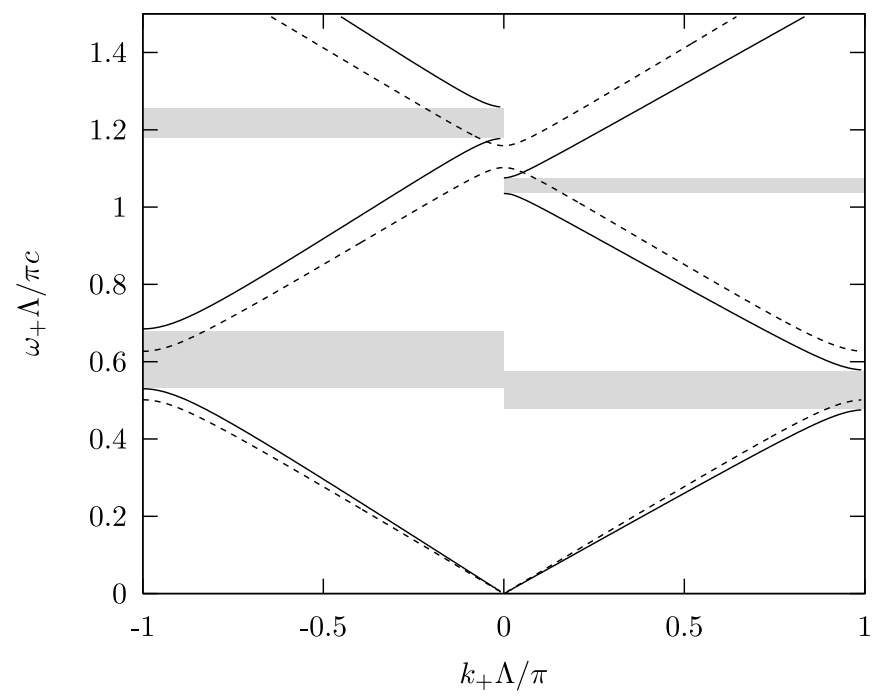

Fig. 2. The dispersion relation $\omega_{+}\left(k_{+}\right)$of left circularly polarized light propagating in an infinite periodic magneto-optical structure in which the magneto-optical modulation is zero, for $B_{0}^{z}=0$ (dashed) and $B_{0}^{z}>0$ (solid). The optical band gaps are in the graph indicated by grey regions. Notice the asymmetric influence of the application of a static magnetic field, clearly discriminating between forward and backward traveling modes. For a right circularly polarized field, the analogous dispersion relation is obtained by using the inversion symmetry property $\omega_{-}\left(k_{-}\right)=\omega_{+}\left(-k_{+}\right)$.

the case of a non-dispersive medium and for the sake of simplicity in the limit of zero modulation of the magneto-optical coefficient, with $\tilde{\gamma}_{+1}=\tilde{\gamma}_{-1}=0$. In the numerical calculation of the band diagram shown in Fig. 2, a value of $M=7$ was used, and in order to clearly illustrate the band gap and effect of the magnetooptical interaction, the refractive index modulation and gyration coefficient were exaggerated compared to a real medium such as, for example, a fiber Bragg grating, in which the frequency width of the band gap typically is of the order of $10^{-4}$ of its center frequency; an explicit formula for the width of the band gap in magnetooptical gratings is given in Sec. 5 .

As shown by the solid graph in Fig. 2, the main impact of the inclusion of magneto-optical interactions is the symmetry-breaking of the dispersion characteristics of forward and backward traveling modes of the electromagnetic field. While the dispersion curve of the forward traveling mode for positive values of $\tilde{\gamma}$ is effectively shifted upwards by the magneto-optical interaction, the dispersion curve of the backward traveling is shifted in the opposite direction. As the optical band gaps as experienced in forward and backward directions of propagation are shifted in opposite directions relative to each other, illustrated by the gray regions in Fig. 2, this implies that the spectral selection and transmission characteristics of the grating can be considerably different in opposite directions of propagation. In particular, for nonlinear optical processes this means that a circularly polarized wave launched in the backward direction will experience a considerably different situation for the 
phase matching in comparison to the same polarization state being launched in the forward direction.

The symmetry breaking as demonstrated here is a property not only intertwined with the magneto-photonic crystals, but also applies to microscopic electronic properties of magneto-optic media in general. In fact, a similar skewness is naturally found for example in the electronic band structure of magnetic semiconductors. ${ }^{31}$

\section{Two Limiting Cases in Wave Propagation}

Before proceeding with actually solving the nonlinear and coupled system of Eq. (13), the solution to the two limiting cases of a linear medium and a spatially non-modulated medium will be discussed. These special cases are important as references for the design process, since the linear optical regime essentially sets the dispersion characteristics necessary for achieving an efficient phase matching, while the case of a longitudinally spatially uniform medium sets the reference level of parametric amplification, against which the figure of merit of the grating-enhanced configuration is judged.

\subsection{Linear regime}

For the case of linear wave propagation the nonlinear source terms are neglected, and the system (13) is considerably simplified by noticing that no backward scattering occur for wave components other than those belonging to the signal wave. The resulting linear coupled equations of motion for the signal wave are straightforward to integrate analytically, to yield the general solution for the forward and backward traveling components as

$$
\begin{aligned}
A_{1}^{\mathrm{f} \pm}(z)= & {\left[G_{1}^{ \pm} \sinh \left(\xi_{ \pm} z\right)+G_{2}^{ \pm} \cosh \left(\xi_{ \pm} z\right)\right] \exp \left(-i \alpha_{ \pm} z\right) } \\
A_{1}^{\mathrm{b} \mp}(z)= & -\left(i / \kappa_{ \pm}^{*}\right)\left\{G_{1}^{ \pm}\left[\alpha_{ \pm} \sinh \left(\xi_{ \pm} z\right)+i \xi_{ \pm} \cosh \left(\xi_{ \pm} z\right)\right]\right. \\
& \left.+G_{2}^{ \pm}\left[\alpha_{ \pm} \cosh \left(\xi_{ \pm} z\right)+i \xi_{ \pm} \sinh \left(\xi_{ \pm} z\right)\right]\right\} \exp \left(i \alpha_{ \pm} z\right)
\end{aligned}
$$

in which the short-hand notation $\xi_{ \pm}=\left(\left|\kappa_{ \pm}\right|^{2}-\alpha_{ \pm}^{2}\right)^{1 / 2}$ was introduced, incorporating the frequency detuning $\alpha_{ \pm}$of the signal as given by Eq. (10), and the propagation coefficient of the waveguiding structure via the coupling coefficient $\kappa_{ \pm}$ as given by Eq. (14). In Eqs. (25), $G_{1}^{ \pm}$and $G_{2}^{ \pm}$are constants of integration determined by the boundary conditions of the grating. The solutions given by Eqs. (25) should not be confused with the dispersion relation as previously obtained by the Bloch theory, as the latter relates to an infinite medium with perfect band gaps, serving so as to discuss the asymptotic limit of phase matching, while the present solutions relate to a medium of finite length. However, in the limit of an infinitely long grating the two cases naturally converge.

By applying the boundary condition that no light is input at the exit end of the grating, the requirement on Eq. (25b) yields $A_{1}^{\mathrm{b} \mp}(L)=0$. By furthermore expressing 
the solutions in terms of the original complex field envelopes by using Eqs. (8), the explicit solutions for the forward and backward traveling components are

$$
\begin{aligned}
E_{\omega_{1}}^{\mathrm{f} \pm} & (x, y, z) \\
= & A_{1}^{\mathrm{f} \pm}(0) F_{ \pm}(x, y)\left[\cosh \left(\xi_{ \pm} z\right)-\frac{\alpha_{ \pm} \cosh \left(\xi_{ \pm} L\right)+i \xi_{ \pm} \sinh \left(\xi_{ \pm} L\right)}{\alpha_{ \pm} \sinh \left(\xi_{ \pm} L\right)+i \xi_{ \pm} \cosh \left(\xi_{ \pm} L\right)} \sinh \left(\xi_{ \pm} z\right)\right] \\
& \quad \times \exp \left(i \beta_{1}^{ \pm} z-i \alpha_{ \pm} z\right), \\
E_{\omega_{1}}^{\mathrm{b}} & (x, y, z) \\
= & \left(i / \kappa_{ \pm}^{*}\right) A_{1}^{\mathrm{f} \pm}(0) F_{ \pm}(x, y)\left[\frac{\alpha_{ \pm} \cosh \left(\xi_{ \pm} L\right)+i \xi_{ \pm} \sinh \left(\xi_{ \pm} L\right)}{\alpha_{ \pm} \sinh \left(\xi_{ \pm} L\right)+i \xi_{ \pm} \cosh \left(\xi_{ \pm} L\right)}\right. \\
& \left.\quad \times\left(\alpha_{ \pm} \sinh \left(\xi_{ \pm} z\right)+i \xi_{ \pm} \cosh \left(\xi_{ \pm} z\right)\right)-\left(\alpha_{ \pm} \cosh \left(\xi_{ \pm} z\right)+i \xi_{ \pm} \sinh \left(\xi_{ \pm} z\right)\right)\right] \\
& \times \exp \left(-i \beta_{1}^{ \pm} z+i \alpha_{ \pm} z\right) .
\end{aligned}
$$

The explicit solutions given by Eqs. (26) fully specify the intra-grating spatial evolution of the optical fields at low intensities, and are highly useful for design purposes. For a grating of geometrical length $L$ the complex-valued linear transmission and reflection coefficients $\tau_{ \pm}=E_{\omega_{1}}^{\mathrm{f} \pm}(L) / E_{\omega_{1}}^{\mathrm{f} \pm}(0)$ and $\rho_{ \pm}=E_{\omega_{1}}^{\mathrm{b} \mp}(0) / E_{\omega_{1}}^{\mathrm{f} \pm}(0)$ of the signal are from Eqs. (26) obtained as

$$
\begin{aligned}
\rho_{ \pm} & =\frac{-i \kappa_{ \pm} \sinh \left(\xi_{ \pm} L\right)}{\left[\alpha_{ \pm} \sinh \left(\xi_{ \pm} L\right)+i \xi_{ \pm} \cosh \left(\xi_{ \pm} L\right)\right]}, \\
\tau_{ \pm} & =\frac{i \xi_{ \pm} \exp \left(i \beta_{1}^{ \pm} L-i \alpha_{ \pm} L\right)}{\left[\alpha_{ \pm} \sinh \left(\xi_{ \pm} L\right)+i \xi_{ \pm} \cosh \left(\xi_{ \pm} L\right)\right]},
\end{aligned}
$$

satisfying the polarization-dependent Stokes relation $\left|\tau_{ \pm}\right|^{2}+\left|\rho_{ \pm}\right|^{2}=1$. Notice that in this regime, the reflection and transmission coefficients seem to be independent of the transverse spatial profile of the waveguiding structure; however, the influence of the waveguiding on the reflection or transmission spectra is always present through the modification of the propagation coefficients $\beta_{1}^{ \pm}$, which in turn effectively shifts the response of the grating in the spectrum as compared to a stratified medium in which no dependence on the transverse coordinates is present.

From Eqs. (27), the stop band corresponding to the band gap as derived from the Bloch theory in Sec. 4 is obtained as the parameter domain in which $\xi_{ \pm}$are real, or

$$
K / 2-\left|\kappa_{ \pm}\right| \leq \beta_{1}^{ \pm} \leq K / 2+\left|\kappa_{ \pm}\right| .
$$

From this, a measure of the spectral width $\Delta \omega_{ \pm}$of the band gap for left/right circular polarization states can be obtained by approximating the propagation coefficients of the signal as $\beta_{1}^{ \pm} \approx \omega_{1} \tilde{n}_{1}\left(1 \pm \tilde{\gamma}_{1} / 2 \tilde{n}_{1}^{2}\right) / c$, giving

$$
\Delta \omega_{ \pm}=\frac{(a \pm b) \omega_{1}}{2 \tilde{n}_{1}\left(1 \pm \tilde{\gamma}_{1} / 2 \tilde{n}_{1}^{2}\right)^{2}} .
$$


Another important figure of merit of the grating is the transmission extinction ratio, which is given as the quote between the minimum transmission at Bragg resonance, at $\omega_{\text {res }}=c K / 2 \tilde{n}_{1}$, and the (unitary) maximum transmission. In a logarithmic (dB) scale, the extinction ratio for left/right circularly polarized modes is obtained from Eqs. (27) as

$$
\begin{aligned}
\xi_{\mathrm{dB}} & \equiv-10 \log _{10}\left(\frac{\left|\tau_{ \pm}\right|_{\max }^{2}}{\left|\tau_{ \pm}\right|_{\min }^{2}}\right) \\
& =-10 \log _{10}\left[1+2 \sinh ^{2}\left(\frac{\pi(a \pm b) L}{8 \tilde{n}\left(1 \pm \tilde{\gamma}_{1} / 2 \tilde{n}_{1}^{2}\right) \Lambda}\right)\right]
\end{aligned}
$$

For strong gratings with moderate magneto-optical modification of the propagation coefficients, this logarithmic extinction ratio is well approximated by

$$
\xi_{\mathrm{dB}} \approx 10 \log _{10} 2-\frac{5 \pi(a \pm b) L}{2 \tilde{n}_{1} \Lambda} \log _{10} e .
$$

In particular, whenever $(a \pm b) L / \tilde{n}_{1} \Lambda \geq 2.7$, corresponding to gratings with an extinction ratio of at least $14.4 \mathrm{~dB}$, a comparison between the exact expression (29) and its approximation (30) reveals that the relative error introduced by the approximation always will be less than one percent.

The signal transmission spectrum given by Eq. (27a) is shown in Fig. 3. For more complicated distributions of the refractive index or magneto-optical gyration coefficient, one can instead discretize the medium into finite elements with the subsequent application of the transfer matrix formalism of magneto-optical Bragg gratings to yield numerical results with high accuracy, in linear as well as nonlinear optical regimes, in the latter for example incorporating optical Kerr-effect and the

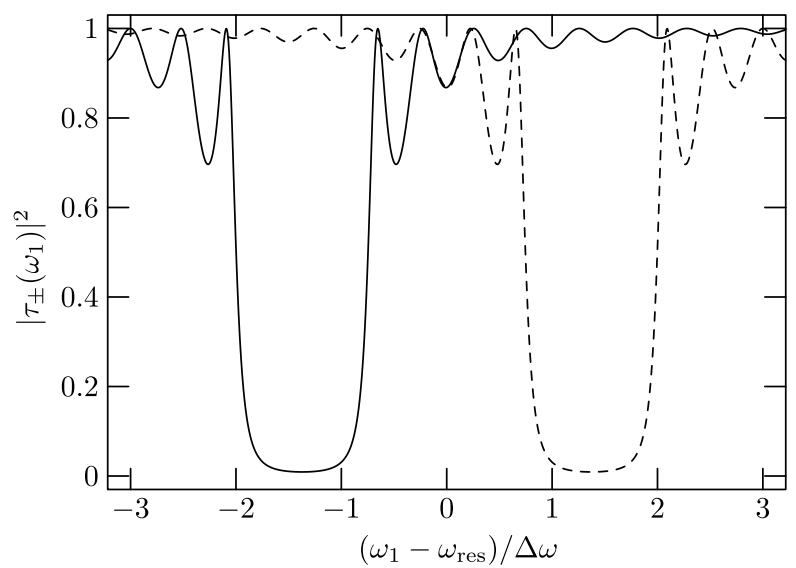

Fig. 3. Transmission of the signal wave as a function of optical frequency $\omega_{1}$, for the incident light being left (solid) and right (dashed) circularly polarized, assuming a zero spatial modulation of the magneto-optical properties, with $b=0$. The frequency is here shifted and scaled in terms of the bias signal resonance frequency $\omega_{\text {res }}=c K / 2 \tilde{n}_{1}$ and transmission band width $\Delta \omega=c K a / 4 \tilde{n}_{1}^{2}$. 
intensity-dependent polarization ellipse rotation as well as their magneto-optical modifications. ${ }^{28,24}$

\subsection{Non-Resonant signal}

As a figure of merit for the grating-assisted parametric conversion, the special case of spatially non-modulated medium is of particular importance. This case has previously been analyzed for magneto-optical parametric down-conversion ${ }^{3,32}$ as well as in optical parametric oscillators, ${ }^{4}$ and we will only quote here the main results in terms of the explicit solutions for the field envelopes, using the same notation as in the present work.

For magneto-optical parametric processes in spatially homogeneous media with $a=b=0$, an exact solution to Eqs. (13) is in the non-depleted pump approximation obtained as ${ }^{3}$

$$
\begin{aligned}
A_{1}^{\mathrm{f} \pm}(z)= & \left\{A_{1}^{\mathrm{f} \pm}(0)\left[\cosh \left(K_{ \pm} z\right)-i\left(\frac{\beta_{ \pm}}{2 K_{ \pm}}\right) \sinh \left(K_{ \pm} z\right)\right]\right. \\
& \left.+i\left(\frac{C_{1}^{ \pm} A_{3}^{\mathrm{f} \mp}(0)}{K_{ \pm}}\right) A_{2}^{\mathrm{f} \pm *}(0) \sinh \left(K_{ \pm} z\right)\right\} \exp \left(i \beta_{ \pm} z / 2\right), \\
A_{2}^{\mathrm{f} \pm}(z)= & \left\{A_{2}^{\mathrm{f} \pm}(0)\left[\cosh \left(K_{ \pm} z\right)-i\left(\frac{\beta_{ \pm}}{2 K_{ \pm}}\right) \sinh \left(K_{ \pm} z\right)\right]\right. \\
& \left.+i\left(\frac{C_{2}^{ \pm} A_{3}^{\mathrm{f} \mp}(0)}{K_{ \pm}}\right) A_{1}^{\mathrm{f} \pm *}(0) \sinh \left(K_{ \pm} z\right)\right\} \exp \left(i \beta_{ \pm} z / 2\right),
\end{aligned}
$$

where

$$
K_{ \pm}=\left(C_{1}^{ \pm} C_{2}^{ \pm *}\left|A_{3}^{\mp}(0)\right|^{2}-\beta_{ \pm}^{2} / 4\right)^{1 / 2}
$$

is the effective gain parameter of the interaction, incorporating the phase mismatch parameter $\beta_{ \pm}$as given by Eq. (11). In the absence of an initial idler wave, the signal amplification is in a medium of geometrical length $L$ obtained from Eqs. (8) and (31a) as

$$
\frac{\left|E_{\omega_{1}}^{\mathrm{f} \pm}(L)\right|^{2}}{\left|E_{\omega_{1}}^{\mathrm{f} \pm}(0)\right|^{2}}=\cosh ^{2}\left(K_{ \pm} L\right)+\frac{\beta_{ \pm}^{2}}{4 K_{ \pm}^{2}} \sinh ^{2}\left(K_{ \pm} L\right)
$$

Whenever the medium is enclosed in optically resonating structures, such as a Fabry-Pérot cavity, exact solutions can also be obtained in terms of Jacobian elliptic functions without employing the non-depleted pump approximation. ${ }^{4}$ However, for our present purpose the simplified solutions obtained within the non-depleted pump approximation are sufficient, since it is within this scope of approximation the final analysis of the equations of motion (13) is to be performed. 


\section{Non-Depleted Pump Approximation}

Whenever the energy transfer rate from the pump field to the signal and idler ones is small as compared to the energy flow of the pump itself, we may in the singly resonant case approximate the non-resonant pump beam as being of constant magnitude across the medium of interaction. Within this approximation, which applies generally whenever

$$
\left|A_{1}^{\mathrm{f} \pm}\right|\left|A_{2}^{\mathrm{f} \pm}\right| \ll\left|A_{3}^{\mathrm{f} \mp}\right|^{2},
$$

the equations of motion (13) for the remaining components of the signal and idler fields take the simpler form

$$
\begin{aligned}
& \frac{\partial A_{1}^{\mathrm{f} \pm}}{\partial z}-\kappa_{ \pm}^{*} A_{1}^{\mathrm{b} \mp} \exp \left(-2 i \alpha_{ \pm} z\right)-i C_{1}^{ \pm} A_{3}^{\mathrm{f} \mp}(0) A_{2}^{\mathrm{f} \pm *} \exp \left(i \beta_{ \pm} z\right)=0, \\
& \frac{\partial A_{1}^{\mathrm{b} \mp}}{\partial z}-\kappa_{ \pm} A_{1}^{\mathrm{f} \pm} \exp \left(2 i \alpha_{ \pm} z\right)=0, \\
& \frac{\partial A_{2}^{\mathrm{f} \pm}}{\partial z}-i C_{2}^{ \pm} A_{3}^{\mathrm{f} \mp}(0) A_{1}^{\mathrm{f} \pm *} \exp \left(i \beta_{ \pm} z\right)=0 .
\end{aligned}
$$

The exponential spatial dependence in Eqs. (33) can be eliminated by taking new field variables $a_{1}^{\mathrm{f} \pm}, a_{1}^{\mathrm{b} \pm}$, and $a_{2}^{\mathrm{f} \pm}$ according to

$$
\begin{aligned}
& A_{1}^{\mathrm{f} \pm}(z)=\left[C_{1}^{ \pm} A_{3}^{\mathrm{f} \mp}(0)\right]^{1 / 2} a_{1}^{\mathrm{f} \pm}(z) \exp \left(-i \alpha_{ \pm} z\right), \\
& A_{1}^{\mathrm{b} \mp}(z)=\left[C_{1}^{ \pm} A_{3}^{\mathrm{f} \mp}(0)\right]^{1 / 2} a_{1}^{\mathrm{b} \mp}(z) \exp \left(i \alpha_{ \pm} z\right), \\
& A_{2}^{\mathrm{f} \pm}(z)=\left[C_{2}^{ \pm} A_{3}^{\mathrm{f} \mp}(0)\right]^{1 / 2} a_{2}^{\mathrm{f} \pm}(z) \exp \left(i\left(\alpha_{ \pm}+\beta_{ \pm}\right) z\right) .
\end{aligned}
$$

This substitution transforms the system given by Eqs. (33) into the autonomous differential equation

$$
\frac{\partial \mathbf{a}_{ \pm}}{\partial z}=\mathbf{A}_{ \pm} \mathbf{a}_{ \pm}
$$

where

$$
\mathbf{a}_{ \pm}(z)=\left(a_{1}^{\mathrm{f} \pm}(z), a_{1}^{\mathrm{b} \pm}(z), a_{2}^{\mathrm{f} \pm *}(z)\right)^{\mathrm{T}}
$$

is a vector containing the normalized and dimensionless envelopes of the interacting fields, and $\mathbf{A}_{ \pm}$is the complex-valued system matrix governing the linear and nonlinear self-coupling and idler cross-coupling between the forward and backward traveling signal modes,

$$
\mathbf{A}_{ \pm}=\left(\begin{array}{ccc}
i(\alpha \pm \delta) & \kappa_{ \pm}^{*} & i \eta_{ \pm} \\
\kappa_{ \pm} & -i(\alpha \pm \delta) & 0 \\
-i \eta_{ \pm} & 0 & i(\alpha \pm \delta+\beta \pm \gamma)
\end{array}\right)
$$

in which

$$
\eta_{ \pm}=\left(C_{1}^{ \pm} C_{2}^{ \pm *}\right)^{1 / 2} A_{3}^{\mathrm{f} \mp}(0)
$$


is the gain parameter describing the strength of the nonlinear energy transfer between the signal and idler,

$$
\alpha=\left(\alpha_{+}+\alpha_{-}\right) / 2 \equiv\left(\beta_{1}^{+}+\beta_{1}^{-}\right) / 2-K / 2
$$

is the bias resonance detuning of the propagation coefficients of the signal,

$$
\delta=\left(\alpha_{+}-\alpha_{-}\right) / 2 \equiv\left(\beta_{1}^{+}-\beta_{1}^{-}\right) / 2
$$

is the differential, magneto-optically induced resonance detuning of the signal,

$$
\beta=\left(\beta_{+}+\beta_{-}\right) / 2
$$

is the bias phase mismatch of the optical parametric process, and finally

$$
\gamma=\left(\beta_{+}-\beta_{-}\right) / 2
$$

is the differential, magneto-optically induced phase detuning of the parametric process, with $\beta_{ \pm}$as previously given by Eq. (11). Notice that while the bias phase mismatch parameter $\beta$ involves the difference between the propagation coefficients of the pump, signal and idler, the magneto-optically determined differential phase detuning acts so as to sum all rotatory strengths of the pump, signal and idler waves. In bulk media, with $\tilde{\gamma}_{1} \ll \tilde{n}_{1}$ and $a^{\prime}=b^{\prime}=0$ in Eqs. (12), with no waveguiding modification to the propagation coefficients, the parameters given in Eqs. (37)-(40) are simply related to the refractive index and gyration coefficients as

$$
\begin{aligned}
\alpha & =\omega_{1} \tilde{n}_{1} / c-K / 2, \quad \delta=\omega_{1} \tilde{\gamma} /\left(2 \tilde{n}_{1} c\right), \\
\beta & =\left(\omega_{3} \tilde{n}_{3}-\omega_{2} \tilde{n}_{2}-\omega_{1} \tilde{n}_{1}\right) / c \\
\gamma & =-\left(\omega_{1} \tilde{\gamma}_{1} / \tilde{n}_{1}+\omega_{2} \tilde{\gamma}_{2} / \tilde{n}_{2}+\omega_{3} \tilde{\gamma}_{3} / \tilde{n}_{3}\right) /(2 c) .
\end{aligned}
$$

As in the case of the grating parameter $\kappa_{ \pm}$, the interaction strengths $\eta_{ \pm}$for orthogonal circular polarization states can always be chosen as real-valued by choosing the initial phases of the pump field, corresponding to a choice of initial orientation of the input polarization ellipse. However, this possibility, contrary to the grating parameter, is dependent on the rotational symmetry of the medium around the axis of wave propagation, that is to say the freedom of choice is opened up by choosing the laboratory $z$-axis as pointing in the $\langle 111\rangle$-direction of the chosen crystal belonging to point-symmetry group $\overline{4} 3 \mathrm{~m}$.

The general solution to the system (35) of linear ordinary differential equations is

$$
\mathbf{a}_{ \pm}(z)=\sum_{k=1}^{3} c_{k_{ \pm}} \boldsymbol{\xi}_{ \pm}^{(k)} \exp \left(\lambda_{ \pm}^{(k)} z\right)
$$

where $\lambda_{ \pm}^{(k)}$ and $\boldsymbol{\xi}_{ \pm}^{(k)}=\left(\xi_{1_{ \pm}}^{(k)}, \xi_{2_{ \pm}}^{(k)}, \xi_{3_{ \pm}}^{(k)}\right)^{\mathrm{T}}$ are the $k$ th non-degenerate respective eigenvalues and eigenvectors of $\mathbf{A}_{ \pm}$, and where $c_{k}^{ \pm}$are constants of integration, determined by boundary conditions. Hence the problem of solving the original system 
of differential equations (13) has been reduced to the problem of finding the eigenvalues, determined by the equation

$$
\operatorname{det}\left(\mathbf{A}_{ \pm}-\lambda_{ \pm}^{(k)} \mathbf{I}\right)=0
$$

and the corresponding eigenvectors, determined by

$$
\left(\mathbf{A}_{ \pm}-\lambda_{ \pm}^{(k)} \mathbf{I}\right) \boldsymbol{\xi}_{ \pm}^{(k)}=\mathbf{0}
$$

where $\mathbf{I}$ denotes the rank-three identity matrix. The complex third degree polynomial equation (42) can, after some straightforward algebra, be solved analytically for the eigenvalues $\lambda_{ \pm}^{(k)}$, in terms of which the corresponding eigenvectors are obtained from Eq. (43) as

$$
\boldsymbol{\xi}_{ \pm}^{(k)}=\left(\begin{array}{c}
(\alpha \pm \delta) \eta_{ \pm}-i \lambda_{ \pm}^{(k)} \eta_{ \pm} \\
-i \kappa_{ \pm} \eta_{ \pm} \\
\left|\kappa_{ \pm}\right|^{2}-(\alpha \pm \delta)^{2}-\lambda_{ \pm}^{(k)} 2
\end{array}\right)
$$

for $k=1,2,3$.

Together with the forms of the variable transformations as used in Eqs. (8) and (34), the eigenvalues and eigenvectors fully determine the general solution for the optical field evolution inside the medium of propagation, in terms of the material properties and optical frequencies. However, in order to determine the coefficients of integration $c_{k}$, which in turn determine the reflected and transmitted field components from given input signal and idler fields $a_{1}^{\mathrm{f}}(0)$ and $a_{2}^{\mathrm{f}}(0)$, boundary conditions must be applied. By evaluating the expression (41) at the beginning $(z=0)$ and end $(z=L)$ of the grating, and furthermore imposing the condition that the backward traveling field component at the end of the grating vanish, the constants of integration $c_{k_{ \pm}}$are obtained as

$$
\left(\begin{array}{c}
c_{1_{ \pm}} \\
c_{2_{ \pm}} \\
c_{3_{ \pm}}
\end{array}\right)=\left(\begin{array}{ccc}
\xi_{1_{ \pm}}^{(1)} & \xi_{1_{ \pm}}^{(2)} & \xi_{1_{ \pm}}^{(3)} \\
\xi_{2_{ \pm}}^{(1)} e_{1_{ \pm}} & \xi_{2_{ \pm}}^{(2)} e_{2_{ \pm}} & \xi_{2_{ \pm}}^{(3)} e_{3_{ \pm}} \\
\xi_{3_{ \pm}}^{(1)} & \xi_{3_{ \pm}}^{(2)} & \xi_{3_{ \pm}}^{(3)}
\end{array}\right)^{-1}\left(\begin{array}{c}
a_{1}^{\mathrm{f} \pm}(0) \\
0 \\
a_{2}^{\mathrm{f} \pm *}(0)
\end{array}\right)
$$

where the notation $e_{k_{ \pm}}=\exp \left(\lambda_{ \pm}^{(k)} L\right)$ was introduced for the sake of algebraic simplicity. The transmitted and reflected field envelopes of the signal $\left(\omega_{1}\right)$ and idler $\left(\omega_{2}\right)$ waves are then from the integration constants and incident field envelopes explicitly obtained as

$$
\left(\begin{array}{c}
a_{1}^{\mathrm{f} \pm}(L) \\
a_{1}^{\mathrm{b} \pm}(0) \\
a_{2}^{\mathrm{f} \pm *}(L)
\end{array}\right)=\left(\begin{array}{ccc}
\xi_{1_{ \pm}}^{(1)} e_{1_{ \pm}} & \xi_{1_{ \pm}}^{(2)} e_{2_{ \pm}} & \xi_{1_{ \pm}}^{(3)} e_{3_{ \pm}} \\
\xi_{2_{ \pm}}^{(1)} & \xi_{2_{ \pm}}^{(2)} & \xi_{2_{ \pm}}^{(3)} \\
\xi_{3_{ \pm}}^{(1)} e_{1_{ \pm}} & \xi_{3_{ \pm}}^{(2)} e_{2_{ \pm}} & \xi_{3_{ \pm}}^{(3)} e_{3_{ \pm}}
\end{array}\right)\left(\begin{array}{c}
c_{1_{ \pm}} \\
c_{2_{ \pm}} \\
c_{3_{ \pm}}
\end{array}\right)
$$

Although being somewhat complex in their algebraic appearance, Eqs. (41)-(46) form an explicit and analytical solution to the signal and idler field evolutions in optical parametric conversion in magneto-optical Bragg gratings. 


\section{Discussion}

As an illustration of the optical parametric process in magneto-optical Bragg gratings, we consider a medium in which only the index of refraction is spatially modulated, with the linear magneto-optical properties being uniform over the extent of the grating, with $b=0$. For simplicity in presenting the ideas, we choose to also neglect the usually small magneto-optical contribution to the nonlinearity, with $\chi_{x x x z}^{\text {eeem }}=0$. However, it should be emphasized that the explicit solutions given by Eqs. (41)-(46) include these terms as well, and that the method of numerical evaluation is identical in these cases. Assuming the nonlinear susceptibility to be roughly one tenth of that of common nonlinear materials possessing a second-order nonlinearity, such as for example $\mathrm{LiNbO}_{2}$, the second-order susceptibility is chosen as $\chi_{x x x}^{\text {eee }}=1.0 \times 10^{-12} \mathrm{~m} / \mathrm{V}$. At an incident pump intensity of $I_{\mathrm{p}}=\left(\varepsilon_{0} c / 2\right)\left|\mathbf{E}_{\omega_{3}}\right|^{2}=100 \mathrm{MW} / \mathrm{cm}^{2}$ this choice corresponds to a nonlinear gain parameter of $\eta=0.3 \mathrm{~cm}^{-1}$.

As for the refractive index modulation, we choose a moderate value of $a=$ $0.9 \times 10^{-4}$ and a bias refractive index $\tilde{n}=1.8$ at a signal wavelength in the order of $1.0 \mu \mathrm{m}$, which gives a linear coupling coefficient of $\left|\kappa_{+}\right|=\left|\kappa_{-}\right|=1.4 \mathrm{~cm}^{-1}$. For a grating of geometrical length $L=3.0 \mathrm{~cm}$, this, from Eq. (30), corresponds to a logarithmic extinction ratio of $\xi_{\mathrm{dB}}=-15.4 \mathrm{~dB}$ in the absence of the optical pump. From Eq. (28) this choice of coupling coefficient furthermore corresponds to a very narrow spectral width of $\Delta \omega / \omega_{1}=2.5 \times 10^{-5}$ of the optical band gap, which at a resonance vacuum wavelength of approximately $1.0 \mu \mathrm{m}$ for the signal corresponds to a spectral width of $\Delta \lambda=0.025 \mathrm{~nm}$. An advantage with employing such small optical band gap is that the magneto-optical interaction strength necessary to achieve tunability of the resonance is moderate. We furthermore assume a moderate bias phase mismatch of $\beta=10.0 \mathrm{~cm}^{-1}$ of the grating, and for the limiting case of a nonmodulated medium as analyzed in Sec. 5, this choice, from Eq. (32), corresponds to a maximum possible amplification of

$$
10 \log _{10}\left(\frac{\left|E_{\omega_{1}}^{ \pm}(L)\right|^{2}}{\left|E_{\omega_{1}}^{ \pm}(0)\right|^{2}}\right)=0.7 \mathrm{~dB},
$$

in the absence of static magnetic fields and in a corresponding homogeneous medium which otherwise possesses the same material parameters.

The logarithmic amplification of a left circularly polarized signal in transmission of a nonlinear grating possessing these material parameters and pump intensity is shown in Fig. 4, as a function of the grating detuning parameter $\alpha$. This graph acts as a design example illustrating optimization of the detuning parameter in designing a proper phase matching scheme of the grating, and in order to determine which regions allow an enhancement over the case of a uniform medium, the corresponding amplification in a non-modulated case is included as well. As shown in the figure, the presence of the optical stop band effectively blocks any signal amplification in the resonant region of the grating. However, at a bias detuning of $\alpha=-4.66 \mathrm{~cm}^{-1}$ the signal amplification is considerably increased, reaching a peak value of $13.8 \mathrm{~dB}$. 


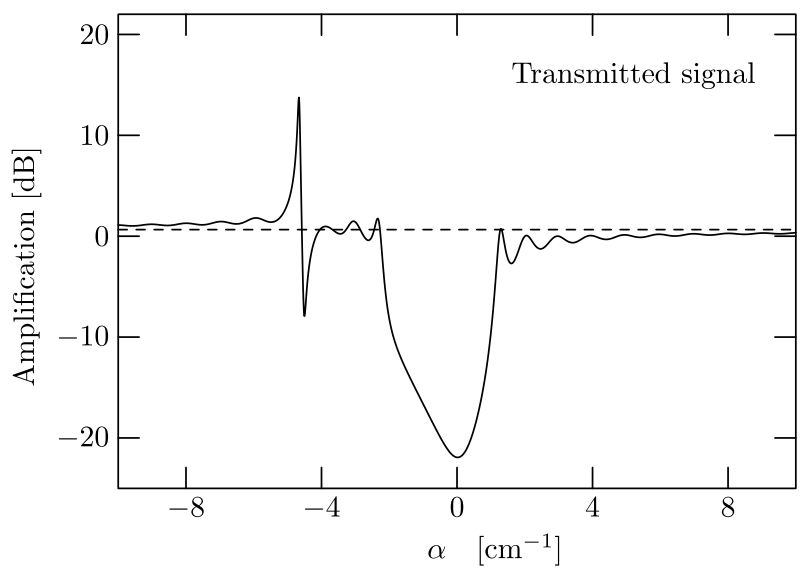

Fig. 4. Amplification $10 \log _{10}\left(\left|E_{\omega_{1}}^{\mathrm{f}+}(L) / E_{\omega_{1}}^{\mathrm{f}+}(0)\right|^{2}\right)$ of a left circularly polarized signal in transmission vs bias detuning $\alpha$ of the grating periodicity relative the signal resonance frequency, in absence of the static magnetic field. The dashed reference line shows the corresponding amplification level of $0.7 \mathrm{~dB}$ in a medium in which the refractive index is spatially uniform. Used parameters are $\beta=10.0 \mathrm{~cm}^{-1}, \gamma=0, \delta=0, \eta=3.0 \mathrm{~cm}^{-1}, \kappa=1.4 \mathrm{~cm}^{-1}$, and geometrical length $L=3.0 \mathrm{~cm}$.

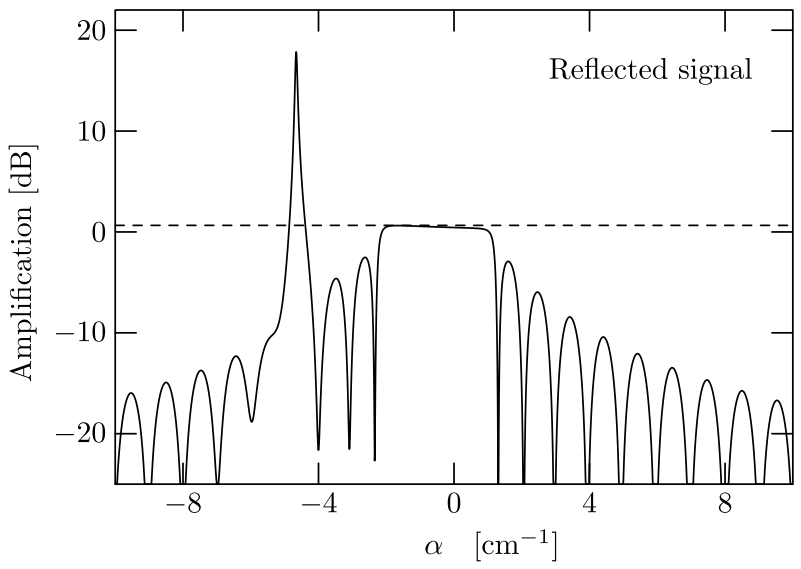

Fig. 5. Logarithmic amplification of the reflected signal vs bias resonance detuning $\alpha$. The dashed reference line shows the corresponding forward amplification in a medium in which the refractive index is spatially uniform. All parameter values used are identical to those used in Fig. 4.

This clearly illustrates the radical impact which can be achieved of the band gap dispersion in grating-assisted phase matching of the parametric amplification. Far from the band gap the amplification asymptotically approaches that of a uniform medium, as expected when the off-resonance transmission reaches unity.

The effect of the band-gap dispersion on the phase matching is not limited only to the transmission mode of amplification; in reflection mode the effect can also be considerable, as shown in Fig. 5. In this case, the signal amplification is even higher, 
reaching a value of $17.9 \mathrm{~dB}$ at the same detuning as for the transmission maximum. As can be seen in the graph, some amplification occurs also in the band gap in reflection mode, though far from ideal levels. However, due to that the test grating as considered here is chosen as not being apodized at the ends, the resulting Gibbs oscillations $^{19}$ at the edges of the band gap are considerable, causing the reflected amplified signal to oscillate heavily with the detuning $\alpha$. This makes the amplification sensitive to variations of the environment of the interaction, such as temperature and strain fluctuations. This potential problem, however, opens up for one of the features brought in by the inclusion of magneto-optical interactions, namely the tunability by means of the strength of the applied static magnetic field, which can be used for compensation of any deviations of parameters of the basic design.

The requirement for the magneto-optical interaction strength to be sufficient for compensation of any drift in the detuning parameter $\alpha$ is that the differential detuning $\delta$ should be able to reach a value corresponding to at least a few widths of the peaks in the Gibbs oscillations, in this case in the order of $1 \mathrm{~cm}^{-1}$. Whether this differential detuning can be reached or not depends on the Verdet coefficient $V$ of the medium and the strength of the applied static magnetic field. For a typical value of $V \equiv\left|\chi_{x y z}^{\text {eem }}\right| \omega /(2 \tilde{n} c)=80 \mathrm{radT}^{-1} \mathrm{~m}^{-1}$ for magneto-optical glasses in a transparent regime, ${ }^{33}$ the maximum achievable differential detuning at a magnetic field of $B_{0}^{z}=1.0 \mathrm{~T}$ is $\delta=0.8 \mathrm{~cm}^{-1}$. Associated with the differential detuning is also the shifting of the response of circularly polarized fields in opposite directions in the spectrum. This effect is shown in Fig. 6 for a linearly polarized input signal

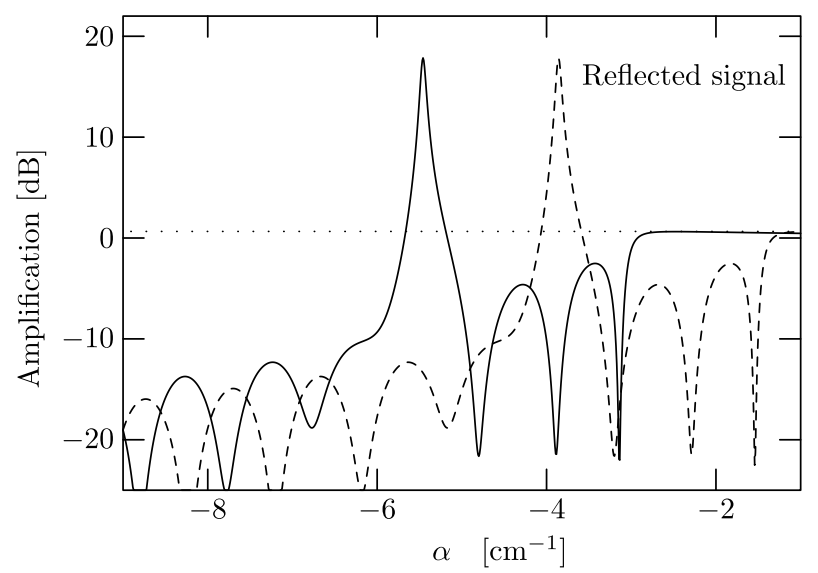

Fig. 6. Logarithmic amplification of the reflected left (solid) and right (dashed) circularly polarized signal vs bias resonance detuning $\alpha$ in the presence of a static magnetic field of $B_{0}^{z}=1.0 \mathrm{~T}$, acting so as to lift the polarization state degeneracy. The dotted reference line shows the corresponding forward amplification in a medium in which the refractive index is spatially uniform. The magnetooptical material parameters were taken as $\delta / B_{0}^{z}=0.8 \mathrm{~cm}^{-1} \mathrm{~T}^{-1}$ and $\gamma / B_{0}^{z}=-2.0 \mathrm{~cm}^{-1} \mathrm{~T}^{-1}$, with all other parameter values identical to those used in Fig. 4. 
and pump. As shown in the figure, the peaks of amplification are clearly resolved between the reflected left and right circular polarization states.

In the grating-assisted parametric amplification as considered here, the periodic modulation of the medium and the associated detuning parameter $\alpha$ clearly have the main roles in achieving a high efficiency, with the magneto-optical interactions providing the means to adjust any imperfections or deviations of the design set by the bias detuning. For instance, by choosing operation at the optimum of amplification in reflection in the absence of static magnetic fields, this naturally means that the amplification of the signal will decrease as the magnetic field sets in, in a symmetrical manner as illustrated in Fig. 7; the point here is rather that the magneto-optical interaction should be employed as a means for compensation whenever environmental changes causes a drift in the optimum amplification, using the tunability as illustrated in Fig. 6.

Associated with the magneto-optical interaction is also the polarization state selectivity in the amplification. By again choosing the case of optimum amplification in the absence of magnetic fields, for which we expect the degenerate case in which left and right circular polarization states act on equal footing, the case of a linearly polarized signal and pump gives a linearly polarized amplified signal. As the magnetic field is switched on, the polarization state of the amplified signal naturally varies with the applied field strength; however, in contrast to the case of the amplitude, the polarization state of the amplified light shows clearly asymmetrical characteristics with respect to the applied static magnetic field, as shown in Fig. 8,

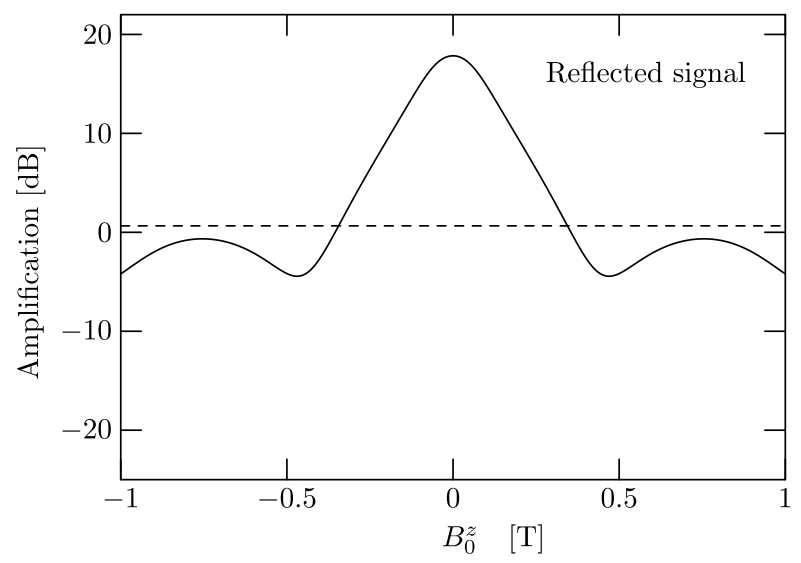

Fig. 7. Logarithmic amplification $10 \log _{10}\left(\left|E_{\omega_{1}}^{\mathrm{b}+}(0)\right|^{2}+\left|E_{\omega_{1}}^{\mathrm{b}-}(0)\right|^{2}\right) /\left(\left|E_{\omega_{1}}^{\mathrm{f}+}(0)\right|^{2}+\left|E_{\omega_{1}}^{\mathrm{f}-}(0)\right|^{2}\right)$ of the reflected signal vs static magnetic field $B_{0}^{z}$, in a configuration with linearly polarized incident signal and pump fields and with the resonance detuning chosen as corresponding to the maximum amplification as in Fig. 5, with $\alpha=-4.66 \mathrm{~cm}^{-1}, \delta / B_{0}^{z}=0.8 \mathrm{~cm}^{-1} \mathrm{~T}^{-1}$, and $\gamma / B_{0}^{z}=-2.0 \mathrm{~cm}^{-1} \mathrm{~T}^{-1}$. The dashed reference line shows the corresponding forward amplification in a medium in which the refractive index is spatially uniform. All other parameter values used are identical to those used in Fig. 4. 


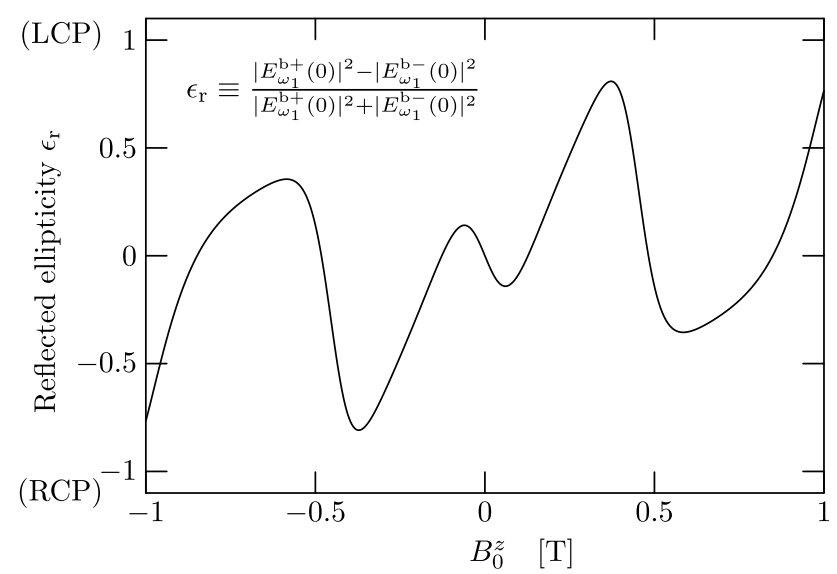

Fig. 8. Normalized ellipticity $\epsilon_{\mathrm{r}}$ of the reflected signal vs static magnetic field $B_{0}^{z}$, with all parameter values identical to those used in Fig. 7.

where the reflected polarization state ellipticity

$$
\epsilon_{\mathrm{r}} \equiv \frac{\left|E_{\omega_{1}}^{\mathrm{b}+}(0)\right|^{2}-\left|E_{\omega_{1}}^{\mathrm{b}-}(0)\right|^{2}}{\left|E_{\omega_{1}}^{\mathrm{b}+}(0)\right|^{2}+\left|E_{\omega_{1}}^{\mathrm{b}-}(0)\right|^{2}}
$$

is mapped against the applied static magnetic field. In this normalized form, $\epsilon_{\mathrm{r}}=-1$ corresponds to right circular, $\epsilon_{\mathrm{r}}=0$ to linear, and $\epsilon_{\mathrm{r}}=1$ to left circular polarization state.

\section{Conclusions}

In conclusion, we have presented an analytical theory of optical parametric interactions in magneto-optical Bragg gratings. The non-reciprocity introduced in the band structure of magneto-optical gratings was discussed in terms of Bloch theory of infinite media, and further linked to cases with gratings of finite extent. Phase matching schemes for optical parametric amplification relying on the dispersion characteristics introduced by a spatial modulation of the linear optical properties of the medium were discussed, and by imposing linear magneto-optical effect onto the phase matching we have shown the symmetrical and asymmetrical impacts on the amplitude and polarization state of the amplified signal, respectively. An advantage with using small modulation amplitudes of the refractive index together with a longer grating is that the effective interaction length of the nonlinear process increases, while also providing for a very narrow optical band gap, which enables a wide magneto-optical tunability at moderate magnetic field intensities also in media with small Verdet constants. 


\section{References}

1. H. Rabin and P. P. Bey, Phys. Rev. 156 (1967) 1010.

2. P. P. Bey and H. Rabin, Phys. Rev. 162 (1967) 794.

3. F. Jonsson and C. Flytzanis, Opt. Lett. 24 (1999) 1514.

4. F. Jonsson and C. Flytzanis, Opt. Lett. 25 (2000) 1249.

5. F. Jonsson and C. Flytzanis, Phys. Rev. Lett. 82 (1999) 1426.

6. N. Bloembergen and A. J. Sievers, Appl. Phys. Lett. 17 (1970) 483.

7. C. Tang and P. Bey, IEEE J. Quantum Electron. QE-9 (1973) 9.

8. A. Yariv and P. Yeh, J. Opt. Soc. Am. 67 (1977) 438.

9. J. Martorell and R. Corbalán, Opt. Comm. 108 (1994) 319.

10. M. J. Steel and N. G. R. Broderick, in Nonlinear Photonic Crystals, eds. R. E. Slusher and B. J. Eggleton (Springer-Verlag, Berlin, 2003), Chap. 10, pp. 221-252.

11. J. D. Jackson, Classical Electrodynamics, 2nd edn. (John Wiley \& Sons, New York, 1975).

12. P. Yeh, J. Opt. Soc. Am. 69 (1979) 742.

13. B. Gralak, S. Enoch and G. Tayeb, J. Opt. Soc. Am. A 17 (2000) 1012.

14. P. N. Butcher and D. Cotter, in The Elements of Nonlinear Optics (Cambridge University Press, New York, 1990), Appendix 3, Table A.3.2.

15. C. Buss et al., Phys. Rev. Lett. 78 (1997) 4123.

16. M. Haddad et al., Appl. Phys. Lett. 73 (1998) 1940.

17. Y. R. Shen, The Principles of Nonlinear Optics (Wiley, New York, 1984).

18. S. Kielich and R. Zawodny, Acta Phys. Pol. A 43 (1973) 579.

19. A. Othonos and K. Kalli, Fiber Bragg Gratings (Artech House, Boston, 1999).

20. L. D. Landau and E. M. Lifshitz, The Classical Theory of Fields, 4th revised english edn. (Butterworth-Heinemann, Oxford, 1975).

21. J. L. Synge and A. Schild, Tensor Calculus (Dover Publications, New York, 1978).

22. L. D. Landau, E. M. Lifshitz and L. P. Pitaevskiı̌, Electrodynamics of Continuous Media, 2nd edn. (Butterworth-Heinemann, Oxford, 1984).

23. F. Jonsson and C. Flytzanis, J. Opt. Soc. Am. B 22 (2005) 293.

24. F. Jonsson and C. Flytzanis, Phys. Rev. Lett. 96 (2006) 063902.

25. A. W. Snyder and J. D. Love, Optical Waveguide Theory (Kluwer Academic Publishers, Boston, 1983).

26. R. März, Integrated Optics — Design and Modeling (Artech House, Boston, 1995).

27. H. Kogelnik, in Guided-Wave Optoelectronics, Springer Series in Electronics and Photonics, 2nd edn., ed. T. Tamir (Springer-Verlag, Berlin, 1990), Chap. 2, pp. 7-88.

28. F. Jonsson and C. Flytzanis, J. Nonl. Opt. Phys. Mater. 13 (2004) 129.

29. C. Kittel, Introduction to Solid State Physics, 6th edn. (John Wiley \& Sons, New York, 1986).

30. K. Sakoda, Optical Properties of Photonic Crystals (Springer-Verlag, Berlin, 2001).

31. C. Xu et al., Phys. Rev. B 68 (2003) 193201.

32. F. Jonsson and C. Flytzanis, J. Opt. A: Pure Appl. Opt. 2 (2000) 299.

33. N. G. Kalugin and G. Wagnière, J. Opt. B: Quantum Semiclass. Opt. 3 (2001) S189. 